\title{
Future SIDIS measurements with a transversely polarised deuteron target at COMPASS
}

\author{
Anna Martin \\ Trieste University and INFN, Trieste, Italy \\ E-mail:anna.martin@ts.infn.it \\ on behalf of the COMPASS Collaboration
}

\begin{abstract}
Since 2005, measurements of Collins and Sivers asymmetries from the HERMES and COMPASS experiments have shown that both the transversity and the Sivers PDFs are different from zero and measurable in semi-inclusive DIS on transversely polarised targets. Most of the data were collected on proton targets, and only small event samples were collected in the early phase of the COMPASS experiment on a deuteron $\left({ }^{6} \mathrm{LiD}\right)$ target and more recently at JLab, on ${ }^{3} \mathrm{He}$. As a consequence, the $d$-quark and the sea-quarks transversity and Sivers PDFs are much more poorly known than those of the $u$-quark. This constitutes an important limitation to the knowledge of the transverse spin and transverse momentum structure of the nucleon. The COMPASS Collaboration has proposed to measure semi-inclusive DIS on transversely polarised deuterons with good accuracy, comparable with that of the existing transverse spin asymmetry data on protons. The experiment will take data in 2021, as soon as the CERN long shut-down 2 will be over, providing measurements which will stay unique for many years to come. In particular, the uncertainties on the $d$-quark transversity PDF is expected to be smaller than the present one by a factor 2 to 4 in the measured $x$ range, and that on the isovector tensor charge of the nucleon will be reduced by a factor of two.
\end{abstract}

XXVII International Workshop on Deep Inelastic Scattering and Related Subjects (DIS2019)

Torino, Italy

April 8-12, 2019 
The partonic structure of the nucleon is presently described by the transverse-momentumdependent distribution functions (TMD PDFs) which generalise the collinear PDFs by taking into account the transverse momentum $k_{T}$ of the parton with respect to the nucleon direction (for reviews, see [1-3]). At leading twist a complete picture of the nucleon requires for each quark flavour eight TMD PDFs, which depend on the parton light-cone momentum fraction $x$, on a characteristic hard scale $Q^{2}$, and on $k_{T}$.

The lepton-nucleon semi-inclusive DIS (SIDIS) is a unique tool to study these new distributions. In particular, when the target nucleon is transversely polarised, five different spin-dependent azimuthal modulations (eight if the leptons are longitudinally polarised, as in the COMPASS case) are expected in the cross-section, and their amplitudes are often indicated as transverse-spin asymmetries (TSA). Some of them are given by the convolution over transverse momenta of TMD PDFs and fragmentation functions (FFs), thus important information on the TMD PDFs can be extracted from a measurement of the SIDIS cross-section.

In this domain the HERMES [4,5] and the COMPASS [6-9] Collaborations have performed pioneering measurements at different beam energies (27 and $160 \mathrm{GeV} / c$ respectively) producing fundamental results. They have shown beyond any doubt that two transverse-spin dependent azimuthal modulations of the produced hadrons, of the type $\sin \left(\phi_{h}+\phi_{S}-\pi\right)$ and $\sin \left(\phi_{h}-\phi_{S}\right)$, are clearly different from zero. The azimuthal angles of the produced hadron transverse momentum $\phi_{h}$ and of the spin direction of the target nucleon $\phi_{S}$ are defined with respect to the lepton scattering plane, in a reference system in which the $\mathrm{z}$ axis is the virtual-photon direction. The amplitude of the first modulation, called Collins asymmetry, is described as a convolution of the transversity function and a newly introduced TMD FF, the Collins FF, $H_{1}^{\perp}$. The amplitude of the second modulation, called the Sivers asymmetry, is described as a convolution between the Sivers function, a TMD PDF, and the usual fragmentation function.

The fact that the Collins and the Sivers asymmetries are non zero on a proton target implies that the Collins FF $H_{1}^{\perp}$ is different from zero, and that both the transversity PDF $h_{1}$ and the Sivers PDF $f_{1 T}^{\perp}$ are different from zero. Independent evidence that the Collins FF is different from zero was provided by the measurement of azimuthal asymmetries in inclusive production of hadron pairs in $e^{+} e^{-} \rightarrow$ hadrons annihilation by the Belle Collaboration [10], and more recently by the BaBar [11] and by the BESIII [12] Collaborations.

By combining the HERMES [4] and the COMPASS [6-8] data with the Belle measurements, first extractions of the Collins FF and of the transversity PDF have been possible. Some recent extractions of the transversity function, from Ref. [13] and [14], are shown in Fig. 1. The trend of the functions is similar to that of the $u$ and $d$ helicity distributions. The accuracy with which they are determined, however, is much lower. From Fig. 1 is is also clear that the uncertainty for the $d$-quark is much larger than that for the $u$-quark. This is due to the fact that most of the existing data have been collected on proton targets, and only few measurements on neutron targets have been performed, which are essential for flavour separation. COMPASS has measured SIDIS using both transversely polarised proton and deuteron targets. On the deuteron, the TSAs are compatible with zero [6], but the accuracy of the measurement is definitely inferior to that of the proton data because of the reduced spectrometer acceptance and running time. More recently data have been collected at much lower energy at JLab on a ${ }^{3} \mathrm{He}$ target, essentially a transversely polarised neutron target. The measured asymmetries $[15,16]$ are also compatible with zero, and the error bars are 
again fairly large.

The measurement of the transversity distributions is particularly important because they provide access to the tensor charges $\delta q$, or $g_{T}^{q}$, which are given by the integral

$$
\delta q\left(Q^{2}\right)=\int_{0}^{1} d x\left[h_{1}^{q}\left(x, Q^{2}\right)-h_{1}^{\bar{q}}\left(x, Q^{2}\right)\right]=\int_{0}^{1} d x\left[h_{1}^{q_{v}}\left(x, Q^{2}\right)\right]
$$

In a non-relativistic quark model, $h_{1}^{q}$ is equal to $g_{1}^{q}$, the helicity distribution, and $\delta q$ is equal to the valence quark $q_{v}$ contribution to the nucleon spin. Knowing the quark tensor charges one can construct the isovector nucleon tensor charge $g_{T}=\delta u-\delta d$, a fundamental property of the nucleon which, together with the vector and axial charges, characterizes the nucleon as a whole. Since many years $g_{T}$ is being calculated with steadily increasing accuracy by lattice QCD $[17,18]$, making even more interesting its experimental determination.

A good knowledge of the tensor charge requires measurements of similar accuracy for $h_{1}^{u_{v}}$ and $h_{1}^{d_{v}}$, namely for deuteron and proton Collins asymmetries. For this reason we have proposed [19] to CERN a new one-year (150 days) measurement scattering the M2 muon beam with $160 \mathrm{GeV} / \mathrm{c}$ momentum on a transversely polarised deuteron target, using the COMPASS spectrometer in the same configuration as for the 2010 run, when the largest proton data set was collected. The main objective of the proposal is to measure the deuteron TSA with adequate accuracy from $x \simeq 0.3$ down to 0.003 and at $Q^{2}$ up to $\simeq 100(\mathrm{GeV} / \mathrm{c})^{2}$, thus our measurement will be complementary to the future JLab12 experiments, like SoLID [21], which will provide new and hopefully precise data on both the proton and the deuteron, but at fairly large $x$-values and smaller $Q^{2}$. The COMPASS measurements will be essential both to evaluate the tensor charges and to access the PDFs of the sea quarks in the short term. In a longer time scale, EIC will possibly supersede the existing and the future measurements, but the new COMPASS contribution will stay there for several years. The proposal has been accepted by CERN, and the experiment will run in 2021, as soon as the second long-shut down of the CERN accelerator complex (LS2) will be over.

In the following, only the case for transversity will be detailed, but it is clear that the new SIDIS deuteron data, will allow to measure all the TSAs on deuteron with accuracy comparable with that of the present proton asymmetries. Thus, when combined with the good precision HER-
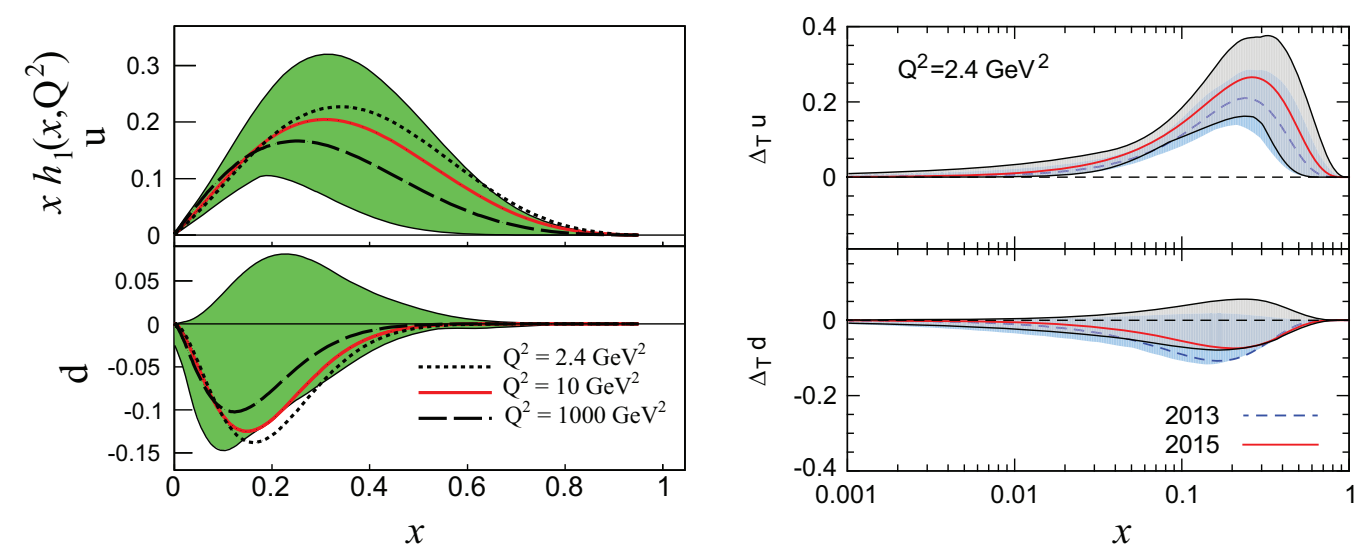

Figure 1: The $u$ and $d$ quark transversity PDFs from recent global fits. The plots are from Ref. [13] (left) and from Ref. [14] (right). 


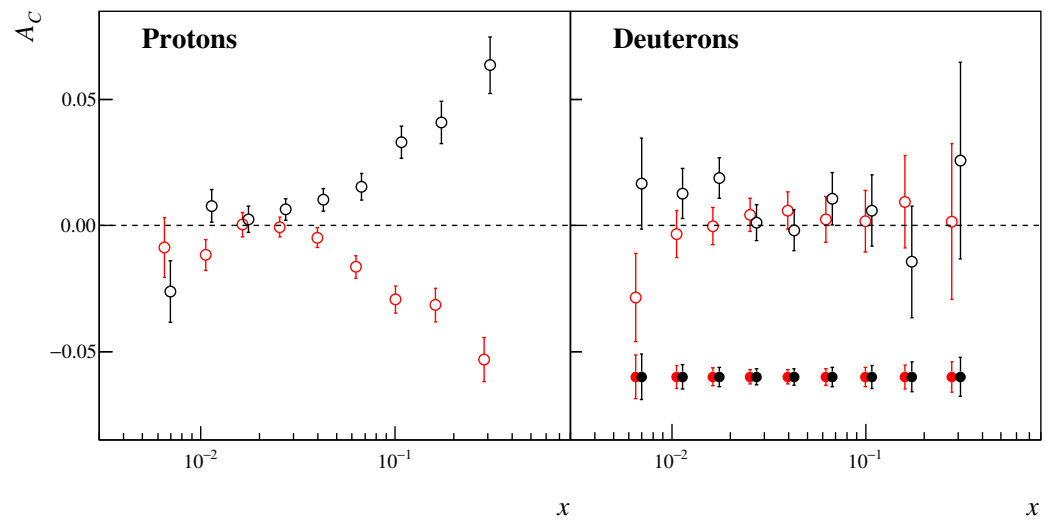

Figure 2: Left plot: the Collins asymmetry $A_{C}$ obtained from the 2010 proton data as a function of $x$. Right plot: the Collins asymmetry obtained from the deuteron runs of 2002, 2003 and 2004. The red (black) points refer to positive (negative) hadrons. The full points at $A_{C}=-0.06$ in the right plot show the extrapolated statistical errors from the 2021 deuteron run.

MES and COMPASS proton data, the new COMPASS deuteron data will allow also the other $u$ and $d$-quark distribution functions to be extracted with comparable accuracy. This is particularly important for the Sivers distribution, the most famous of the TMD PDFs.

In one year of data taking in the conditions of the 2010 proton run we expect the number of reconstructed SIDIS events to be the same as in 2010, since target material densities and packing factors are almost identical for ${ }^{6} \mathrm{LiD}$ and $\mathrm{NH}_{3}$. The statistical uncertainties of the measured TSAs in the future deuteron run will thus just be the statistical uncertainties of the 2010 proton data scaled by the ratio

$$
r=\frac{F O M_{p}}{F O M_{d}}=\frac{f_{p} P_{p}}{f_{d} P_{d}}=\frac{0.155 \cdot 0.80}{0.40 \cdot 0.50}=0.62
$$

where $F O M_{x}$ are the figures of merit of the polarised targets, $f_{x}$ are the dilution factors of the target material, and $P_{x}$ are the proton or deuteron polarisations.

The statistical errors of the transverse spin asymmetries will be smaller by a factor of 0.62 than those of the proton. The projected errors for the deuteron Collins asymmetries are shown as closed points in the right plot of Fig. 2. We neglect the systematic errors which were estimated to be at most 0.5 times the statistical errors in the 2010 data, and are expected to be of the same order in the future run.

In order to quantify the gain in statistical uncertainty in $h_{1}^{u_{v}}$ and $h_{1}^{d_{v}}$, we have extracted twice the $u$ - and $d$-quark transversity, the first time using the existing COMPASS deuteron data, and a second time using for the statistical errors the projections of Fig. 2. In both cases all the existing proton data from HERMES and COMPASS have been used. The values of the transversity distributions have been extracted point by point following the procedure of Ref. [20].

The values of $x h_{1}^{u_{v}}$ and $x h_{1}^{d_{v}}$ obtained using all proton and deuteron existing data are shown in the left panel of Fig. 3. The right panel shows the transversity distributions when using the 2021 

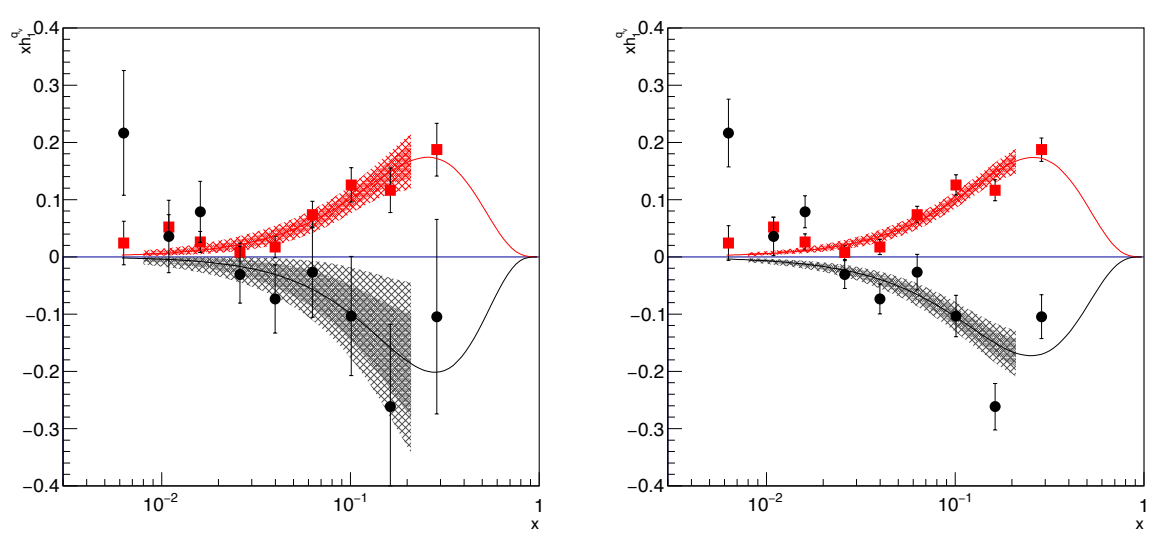

Figure 3: Values for $x h_{1}^{u_{v}}$ (red squares) and $x h_{1}^{d_{v}}$ (black circles) extracted from the measured asymmetries. Left plot: existing deuteron data uncertainties. Right plot: 2021deuteron data uncertainties . Also shown, for both cases, are the $68 \%$ and $90 \%$ confidence bands.

projections for the deuteron uncertainties. In both plots the bands are the $68 \%$ and $90 \%$ confidence bands obtained from 80000 replicas.

To evaluate the tensor charge the transversity PDFs for the valence quarks have to be integrated over $x$ from 0 to 1 . To avoid any possible bias, we prefer not to use parametric functions for the transversity PDFs, and simply integrate numerically the valence quark transversity values, obtained as described previously, in the measured $x$ range only.

The values of $\delta u, \delta d$ and $g_{T}$ in the selected $x$ range are given in Table 1, both for the present uncertainties and using the projected errors for the deuteron. The statistical uncertainties are evaluated by adding up the errors of the measurements in the various $x$-bins, and, for $g_{T}$, taking into account the correlation coefficients of the $u$ - and $d$-quark transversity which become close to zero for the projected deuteron errors. For different reasons, the first $(0.003<x<0.008)$ and the ninth $(0.21<x<0.7) x$-bins give fairly large contributions to the uncertainty of the tensor charge, therefore in the following we integrate the transversity PDFs over the central seven bins $(0.008<x<0.21)$, skipping the first and the last.

The reduction of the uncertainties on the integral of the $d$-quark transversity when the future measurement is taken into account is considerable, from 0.108 to 0.040 . This is the most important outcome we expect from the new run we propose. However, also the knowledge of the $u$-quark transversity integral improves. For the tensor charge $g_{T}$ the uncertainty is reduced by a factor of two.

To summarize, JLab12 will provide very accurate measurements for $g_{T}$ in the region $0.1<$ $x<0.7$. However, using only those data and the existing data, the evaluation of the tensor charge 


\begin{tabular}{|l|c|c|c|}
\hline & $\delta u=\int d x h_{1}^{u_{v}}(x)$ & $\delta d=\int d x h_{1}^{d_{v}}(x)$ & $g_{T}=\delta u-\delta d$ \\
\hline present & $0.201 \pm 0.032$ & $-0.189 \pm 0.108$ & $0.390 \pm 0.087$ \\
projected & $0.201 \pm 0.019$ & $-0.189 \pm 0.040$ & $0.390 \pm 0.044$ \\
\hline
\end{tabular}

Table 1: Truncated tensor charges from all existing proton and deuteron data ("present") and with the projected uncertainties for the deuteron data ("projected"). The integration runs over the range $0.008<x<$ 0.21 .

would be affected by the error of the integration between 0 and 0.1 , and the result would anyhow be model dependent. The relevance of the new deuteron COMPASS data is that, when combined with the existing proton data, they will allow to measure the truncated $g_{T}$ with an uncertainty of \pm 0.044 . in the range $0.008<x<0.21$, thus reducing considerably the systematic uncertainty on $g_{T}$ due to the extrapolation to $x=0$.

\section{References}

[1] V. Barone et al., Prog. Part. Nucl. Phys. 65 (2010) 267.

[2] C. A. Aidala et al., Rev. Mod. Phys. 85 (2013) 655.

[3] H. Avakian et al., Eur. Phys. J. A52 (2016) 150, [Erratum: Eur. Phys. J. A52 No.6 (2016) 165].

[4] HERMES Collaboration, A. Airapetian et al., Phys. Lett. B693 (2010) 11.

[5] HERMES Collaboration, A. Airapetian et al., Phys. Rev. Lett. 103 (2009) 152002.

[6] COMPASS Collaboration, E. S. Ageev et al., Nucl. Phys. B765 (2007) 31.

[7] COMPASS Collaboration, M. G. Alekseev et al., Phys. Lett. B692 (2010) 240.

[8] COMPASS Collaboration, C. Adolph et al., Phys. Lett. B717 (2012) 376.

[9] COMPASS Collaboration, C. Adolph et al., Phys.Lett. B717 (2012) 383.

[10] Belle Collaboration, R. Seidl et al., Phys. Rev. D78 (2008) 032011, [Erratum: Phys. Rev. D86 (2012) 039905].

[11] BaBar Collaboration, J. P. Lees et al., Phys. Rev. D 90 (2014) no.5, 052003.

[12] BESIII Collaboration, M. Ablikim et al., Phys. Rev. Lett. 116 (2016) no.4, 042001.

[13] Z.-B. Kang et al., Phys. Rev. D93 (2016) 014009.

[14] M. Anselmino et al., Phys. Rev. D92 (2015) 114023.

[15] Jefferson Lab Hall A, X. Qian et al., Phys. Rev. Lett. 107 (2011) 072003.

[16] Jefferson Lab Hall A, Y. X. Zhao et al., Phys. Rev. C90 (2014) 055201.

[17] J.-W. Chen et al., Nucl. Phys. B911 (2016) 246.

[18] C. Alexandrou et al., Phys. Rev. D 98 (2018) no.9, 091503.

[19] COMPASS Collaboration, d-Quark Transversity and Proton Radius, Addendum to the COMPASS-II Proposal, CERN-SPSC-2017034 SPSC-P-340-ADD-1, April 5, 2018.

[20] A. Martin et al., Phys. Rev. D91 (2015) 014034.

[21] Z. Ye et al., Phys. Lett. B767 (2017) 91. 